\title{
Atividade eletrocatalítica de um filme de ormosil híbrido contendo ácido fosfomolíbdico para o herbicida prometon
}

\author{
Electrocatalytic activity of a hybrid ormosil \\ film containing phosphomolybdic acid toward \\ prometon herbicide
}

\author{
Victória de Oliveira Margarido ${ }^{1}$, Julia Helena de Paula ${ }^{1}$, \\ Kelly Roberta Francisco ${ }^{1}$, Adriano Lopes de Souza ${ }^{1}$,
}

\footnotetext{
${ }^{1}$ Departamento de Ciências da Natureza, Matemática e Educação, Universidade Federal de São Carlos (UFSCar), campus Araras, Rodovia Anhanguera, Km 174, PO Box 153, CEP: 13604-900, Araras, SP, Brasil.

e-mail: victoriamargarido@gmail.com,julia_depaula@hotmail.com,kfrancisco@ufscar.br, adrianosouza@ufscar.br
}

\section{RESUMO}

Sílica organicamente modificada (abreviada por ormosil, em virtude da expressão inglesa "organically modified silicate") pode ser empregada para imobilizar espécies químicas de interesse para aplicações como em sensores e dispositivos ópticos. Polioxometalatos (POMs) podem ser imobilizados aos poros da rede de ormosil para investigação de suas propriedades eletroativas uma vez que os mesmos são extensamente utilizados como eletrocatalisadores. Entretanto, poucos estudos exploraram atividades eletrocatalíticas de POMs imobilizados em redes de ormosil para herbicidas triazínicos, os quais são extensamente usados na agricultura e no cultivo de grãos e de cana-de-açúcar. Este trabalho descreve a atividade eletrocatalítica de um filme híbrido formado por uma sílica organicamente modificada (ormosil) por 3-cianopropiltrietóxissilano contendo o POM, ácido fosfomolíbdico, para o herbicida triazínico prometon. Experimentos de voltametria cíclica e de voltametria de pulso diferencial foram realizados usando como eletrólito suporte uma solução aquosa de ácido sulfúrico pH 1,0 e mostraram que o filme do ormosil híbrido depositado sobre eletrodo de ouro foi capaz de eletroreduzir prometon na faixa de concentração de $3,6 \times 10^{-6}$ a $18 \times 10^{-6} \mathrm{~mol} \mathrm{~L}^{-1}$. O potencial onde ocorreu a eletroredução do herbicida foi em torno de $0,0 \mathrm{~V}$ vs. $\mathrm{Ag} / \mathrm{AgCl}$, um valor menor do que aqueles encontrados na literatura como por exemplo $+0,75 \mathrm{~V}$ ou $-1,0 \mathrm{~V}$ vs. $\mathrm{Ag} / \mathrm{AgCl}$. Com esses resultados, o filme aqui produzido pode ser usado para novas aplicações de interesse ambiental.

Palavras-chave: prometon, ácido fosfomolíbdico, sílica organicamente modificada, ormosil.

\begin{abstract}
Organically modified silicate (whose abbreviation is ormosil) can be used to immobilize chemical species of interest for applications such as sensors and optical devices. Polyoxometalates (POMs) can be immobilized to the pores of the ormosil network to investigate their electroactive properties since they are widely used as electrocatalysts. However, few studies have explored the electrocatalytic properties of immobilized POMs in ormosil networks toward triazinic herbicides, which are extensively used in agriculture in the cultivation of grains and sugar cane. The work reported here describes the electrocatalytic activity of a hybrid film formed by an organically modified silica (ormosil) with 3-cyanopropyltriethoxysilane containing a POM, phosphomolybdic acid, toward prometon, a triazinic herbicide. Cyclic voltammetry and differential pulse voltammetry experiments were performed using as supporting electrolyte an aqueous solution of sulfuric acid at $\mathrm{pH} 1,0$ and showed that this hybrid ormosil film deposited on gold electrode electroreduced prometon in a concentration range of $3,6 \times 10^{-6}$ to $18 \times 10^{-6} \mathrm{~mol} \mathrm{~L}^{-1}$. The potential value where the herbicide reduction ocurred was around $0,0 \mathrm{~V}$ vs. $\mathrm{Ag} / \mathrm{AgCl}$, lower than those found in the literature as for example $+0,75 \mathrm{~V}$ or $-1,0 \mathrm{~V}$ vs. $\mathrm{Ag} / \mathrm{AgCl}$. With these features, the resulting film can be used for novel applications of environmental interest.
\end{abstract}

Keywords: prometon, phosphomolybdic acid, organically modified silicate, ormosil. 


\section{INTRODUÇÃO}

O uso de alcóxidos de silício como materiais precursores para o processo sol-gel tem contribuído enormemente para a produção de materiais híbridos orgânico-inorgânicos multifuncionais $[\underline{1}, \underline{2}]$. Nesta estratégia, quando pelo menos um dos alcóxidos de silício possui uma funcionalidade orgânica de interesse, ocorre a formação de uma sílica (ou silicato) organicamente modificada cuja abreviação é "ormosil" (da expressão em inglês organically modified silicate). $\mathrm{O}$ ormosil tem os poros da sua rede modificados pela funcionalidade orgânica do respectivo alcóxido de silício escolhido na etapa de síntese. Esses poros da rede de ormosil são formados durante as reações de hidrólise e condensação dos alcóxidos de silício [프, $\underline{4}$.

Espécies químicas de interesse podem ser incorporadas aos poros do ormosil para as mais diversas finalidades como dispositivos ópticos e sensores, por exemplo [5-7]. Quando complexos metálicos ou óxidos de metais de transição são aprisionados nos poros, temos a produção dos chamados materiais híbridos orgânico-inorgânicos. Polioxometalatos (POMs) são exemplos de clusters metál-óxido de tamanho nanométrico que já foram utilizados para a produção de materiais híbridos orgânico-inorgânicos para aplicações na área de sensores eletroquímicos e materiais fotoativos/fotocrômicos [-11]. POMs são compostos extensamente utilizados para estas finalidades em virtude de permanecerem íntegros mesmo após sucessivas reações de transferência eletrônica [12].

Apesar destas contribuições já terem sido reportadas na literatura, poucos trabalhos investigaram as propriedades eletrocatalíticas de POMs imobilizados na forma de materiais híbridos para compostos triazíni$\cos [13,14]$, que são comumente utilizados como herbicidas e podem levar à contaminação da água [15]. Um composto triazínico é aquele que possui um anel N-heterocíclico aromático de 6 membros com átomos de carbono e de nitrogênio alternados em sua estrutura. Um representante desta classe é o herbicida prometon cuja fórmula estrutural está representada na figura 1. Há poucos relatos na literatura sobre oxidação ou redução eletrocatalítica de prometon e não há trabalhos publicados sobre esses processos envolvendo POMs. Prometon tem sido identificado e/ou quantificado principalmente por cromatografia líquida acoplada à espectrometria de massas [16], por extração de fase sólida [17] e por espectroscopia de fluorescência [18].<smiles>COc1nc(NC(C)C)nc(NC(C)C)n1</smiles>

Figura1: Fórmula estrutural do prometon.

Neste trabalho, a atividade eletrocatalítica de um ormosil híbrido de 3-cianopropiltrietóxissilano (CPTS), tetraetilortossilicato (TEOS) e um POM do tipo Keggin, ácido fosfomolíbdico (HPMo), para o herbicida prometon foi investigada. HPMo é constituído por octaedros $\mathrm{MO}_{6}$ agrupados na forma de tríades $\mathrm{M}_{3} \mathrm{O}_{13}$ ao redor do tetraedro central $\mathrm{PO}_{4}$. O ormosil híbrido depositado em eletrodo de ouro na forma de um filme fino pelo método dip coating teve suas propriedades eletrocatalíticas testadas para prometon através das técnicas de voltametria cíclica e de voltametria de pulso diferencial. Espectroscopia de Absorção na região do infravermelho foi a técnica usada para caracterizar o ormosil híbrido na forma de pó.

\section{MATERIAIS E MÉTODOS}

\subsection{Materiais}

Ácido fosfomolíbdico hidratado 99,9\% (HPMo), tetraetilortosilicato 98\% (TEOS), 3cianopropiltrietóxisilano 98\% (CPTS) e prometon Pestanal ${ }^{\circledR}$ Padrão Analítico foram adquiridos da empresa Sigma Aldrich (EUA) e utilizados sem purificação prévia. A água deionizada utilizada para o preparo das soluções dos herbicidas tinha resistividade de $18,2 \mathrm{M} \Omega \mathrm{cm}$. Ácido sulfúrico $98 \%$ e acetona $99,5 \%$ foram adquiridos da empresa Synth (Diadema-SP) e, também, usados sem nenhum tipo de tratamento específico. Nitrogênio comercial foi adquirido da empresa Oxi Araras. 


\subsection{Preparo do ormosil de CPTS e HPMo e deposição do filme híbrido sobre eletrodo de ouro}

A produção do ormosil descrita aqui seguiu o mesmo procedimento reportado em [14]. Em um béquer de polipropileno foram adicionados $25 \mathrm{~mL}$ de acetona juntamente com $58 \mu \mathrm{L}$ de TEOS $(0,26 \mathrm{mmol}), 59 \mu \mathrm{L}$ de CPTS $(0,25 \mathrm{mmol})$ e $14 \mu \mathrm{L}$ de água deionizada $(0,78 \mathrm{mmol})$. Após 10 minutos de agitação magnética, uma solução contendo $69 \mathrm{mg}(0,038 \mathrm{mmol})$ de HPMo em $25 \mathrm{~mL}$ de acetona foi adicionada à mistura anterior e a agitação magnética permaneceu por mais 15 minutos.

Lâminas de ouro de $3 \mathrm{~cm}^{2}$ foram usadas como substratos na deposição do filme híbrido pelo método dip coating usando um elevador lateral ND-R Rotary Dip Coater da Nadetech Innovations. As velocidades de imersão e de retirada do substrato na solução do ormosil foram de $150 \mathrm{~mm} \mathrm{~min}^{-1}$ e a cada etapa de retirada, um tempo de 60s era destinado à secagem ao ar. No total, 20 ciclos de imersão/emersão foram realizados e o filme só foi submetido aos experimentos eletroquímicos após $12 \mathrm{~h}$ de secagem.

Após o preparo dos filmes, a mistura do ormosil híbrido de CPTS e HPMo foi mantida em repouso até a evaporação completa do solvente para que material na forma de pó (denominado de xerogel) fosse obtido. Este foi reservado e utilizado para a caracterização espectroscópica.

\subsection{Experimentos Eletroquímicos}

Os experimentos de voltametria cíclica e de voltametria de pulso diferencial foram realizados usando uma célula eletroquímica convencional de $25 \mathrm{~mL}$ e 3 eletrodos, onde o eletrodo de trabalho foi a lâmina de ouro modificada com o filme híbrido de CPTS e HPMo, o eletrodo de referência foi um eletrodo de $\mathrm{Ag} / \mathrm{AgCl}$ e o contra-eletrodo foi uma lâmina de platina de $1 \mathrm{~cm}^{2}$. O equipamento utilizado foi um Potenciostato PGSTAT101 da Metrohm e o eletrólito suporte empregado nas análises foi uma solução aquosa de ácido sulfúrico $\mathrm{pH} \approx 1,0$. O uso desse meio ácido nas análises é justificado pela decomposição do HPMo em valores de $\mathrm{pH}$ maiores que 1,5 [19]. Previamente aos experimentos eletroquímicos, a solução do eletrólito suporte era deaerada com nitrogênio comercial por 10 minutos. Após esse tempo, o fluxo do gás era mantido acima da solução do eletrólito suporte na célula eletroquímica para manter a atmosfera inerte durante os experimentos. A área geométrica dos eletrodos era de aproximadamente $1 \mathrm{~cm}^{2}$ e apenas soluções recentemente preparadas do herbicida prometon (usando como solvente a solução aquosa de ácido sulfúrico $\mathrm{pH}$ 1,0) foram empregadas nas análises. Cada experimento eletroquímico foi realizado em triplicata.

\subsection{Análise Espectroscópica}

O pó do ormosil seco e triturado foi usado no preparo de uma pastilha de $\mathrm{KBr}$ e a mesma encaminhada para análise em um espectrômetro de absorção na região do infravermelho com Transformada de Fourier (FTIR) modelo Tensor II da Bruker.

\section{RESULTADOS E DISCUSSÃO}

A Figura 2A mostra os voltamogramas cíclicos nas velocidades de varredura de $10 \mathrm{mV} \mathrm{s}^{-1}, 20 \mathrm{mV} \mathrm{s}^{-1}$ e 50 $\mathrm{mV} \mathrm{s}^{-1}$ para o filme híbrido do ormosil de CPTS e HPMo depositado sobre eletrodo de ouro. O eletrólito suporte usado nesses experimentos foi uma solução aquosa de ácido sulfúrico com pH 1,0. Cada um dos três processos eletroquímicos observados corresponde a uma reação química para o HPMo envolvendo dois elétrons cada uma, conforme relatado por TANAKA et al. [20]. Os potenciais formais $\left(\mathrm{E}^{0^{\prime}}\right)$ obtidos para os três

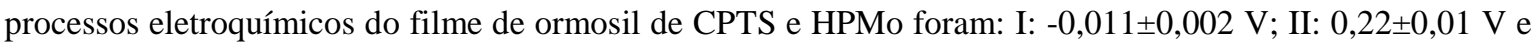
III: $0,34 \pm 0,01 \mathrm{~V}$ vs. $\mathrm{Ag} / \mathrm{AgCl}$. Os valores de $\mathrm{E}^{0^{\prime}}$ foram obtidos a partir da seguinte expressão: $\mathrm{E}^{0^{\prime}}=\left(\mathrm{E}_{\mathrm{pa}}+\mathrm{E}_{\mathrm{pc}}\right) / 2$, onde $\mathrm{E}_{\mathrm{pa}}$ é o potencial de pico anódico e $\mathrm{E}_{\mathrm{pc}}$ é o potencial de pico catódico. Recentemente, nosso grupo de pesquisa encontrou valores muito próximos de $\mathrm{E}^{0^{\prime}}$ para esse filme híbrido de CPTS e HPMo [14]. Esses valores também foram similares aos reportados na literatura nos trabalhos de Li e colaboradores para HPMo imobilizado em nanotubos de carbono [21] e de Rahman e colaboradores para HPMo ancorado em um filme de aminopropiltrietóxissilano [22]. O encarte da figura 2A mostra a variação da densidade de corrente (J) para o segundo processo eletroquímico em função das velocidades de varredura. Uma vez que essa variação é linear e que as diferenças entre os potenciais de pico catódico e anódico permanecem próximas de zero $\left(\Delta \mathrm{E}_{\mathrm{p}} \approx 0\right)$ para cada um dos processos, pode-se afirmar que HPMo está imobilizado na superfície do eletrodo [23]. 


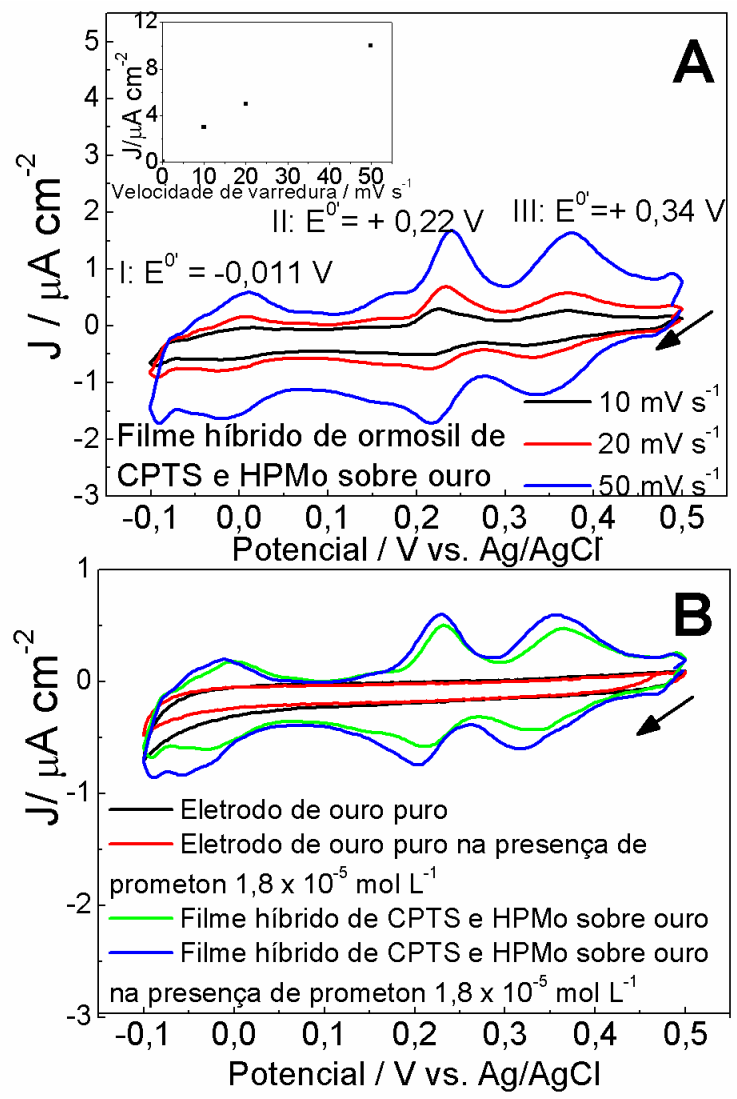

Figura 2: A) Voltamogramas cíclicos nas velocidades de varredura de $10 \mathrm{mV} \mathrm{s}^{-1}, 20 \mathrm{mV} \mathrm{s}^{-1}$ e $50 \mathrm{mV} \mathrm{s}^{-1}$ para o filme híbrido do ormosil de CPTS e HPMo sobre eletrodo de ouro. O eletrólito suporte utilizado foi uma solução aquosa de ácido sulfúrico $\mathrm{pH}$ 1,0. B) Voltamogramas cíclicos na ausência e na presença de uma solução aquosa de prometon 1,8 $\mathrm{x}$ $10^{-5} \mathrm{~mol} \mathrm{~L}^{-1}$ em meio ácido ( $\left.\mathrm{pH} 1,0\right)$ para o eletrodo de ouro puro e para o eletrodo de ouro modificado com o filme híbrido do ormosil de CPTS e HPMo. A velocidade de varredura utilizada foi de $20 \mathrm{mV} \mathrm{s}^{-1}$. O encarte mostra a variação da densidade de corrente $(\mathrm{J})$ em função das velocidades de varredura para o segundo processo eletroquímico.

A figura 2B mostra os voltamogramas cíclicos para o eletrodo de ouro puro e para o eletrodo de ouro modificado com o filme híbrido do ormosil de CPTS e HPMo na ausência e na presença de uma solução aquosa de prometon $1,8 \times 10^{-5} \mathrm{~mol} \mathrm{~L}^{-1}$ em meio ácido $(\mathrm{pH} 1,0)$ na velocidade de varredura de $20 \mathrm{mV} \mathrm{s}^{-1}$. Nota-se que para o eletrodo de ouro puro, não há indícios de que houve oxidação ou redução do herbicida na janela de potencial utilizada. Para o eletrodo modificado com o filme híbrido, há um deslocamento dos processos eletroquímicos do HPMo para menores valores de potencial na presença de prometon e um aumento nas intensidades de densidade de corrente para os dois processos eletroquímicos mais positivos. Para o processo eletroquímico mais negativo, a densidade de corrente na presença de prometon permanece aproximadamente constante e há um aumento na densidade de corrente de redução. Essa mesma tendência é observada na figura 3A, onde os voltamogramas cíclicos para o filme híbrido de CPTS e HPMo obtidos na velocidade de varredura de $20 \mathrm{mV} \mathrm{s}^{-1}$ em meio ácido $(\mathrm{pH} \mathrm{1,0)} \mathrm{e} \mathrm{em} \mathrm{diferentes} \mathrm{concentrações} \mathrm{de} \mathrm{prometon} \mathrm{estão} \mathrm{exibidos.}$

A figura 3B mostra voltamogramas de pulso diferencial para o filme híbrido de CPTS e HPMo em meio ácido ( $\mathrm{pH} \mathrm{1,0)}$ para as mesmas concentrações de prometon reportadas na figura $3 \mathrm{~A}$. Nota-se que para o pico mais negativo, há um ligeiro deslocamento para menor valor de potencial seguido de um aumento da densidade de corrente de redução à medida que as alíquotas de prometon vão sendo adicionadas à célula eletroquímica. Tal comportamento é interpretado como sendo atribuído à redução eletroquímica do prometon pelo HPMo ancorado no filme de ormosil com CPTS no potencial em torno de 0,0 vs. Ag/AgCl. Para os outros dois picos de redução, tal perfil não é observado com clareza. A eletroredução de outro herbicida triazínico, a ametrina, por HPMo incorporado no filme híbrido do ormosil de CPTS já foi relata em um trabalho prévio do nosso grupo de pesquisa [14]. OLIVEIRA-BRETT e SILVA [24] verificaram a oxidação de prometon a $+0,75 \mathrm{~V}$ vs. $\mathrm{Ag} / \mathrm{AgCl}$ através de voltametria de pulso diferencial usando um eletrodo de pasta de carbono modificado com DNA em pH 4,5 em solução tampão acetato. Tal pico foi associado à interação do herbicida com guanina. 

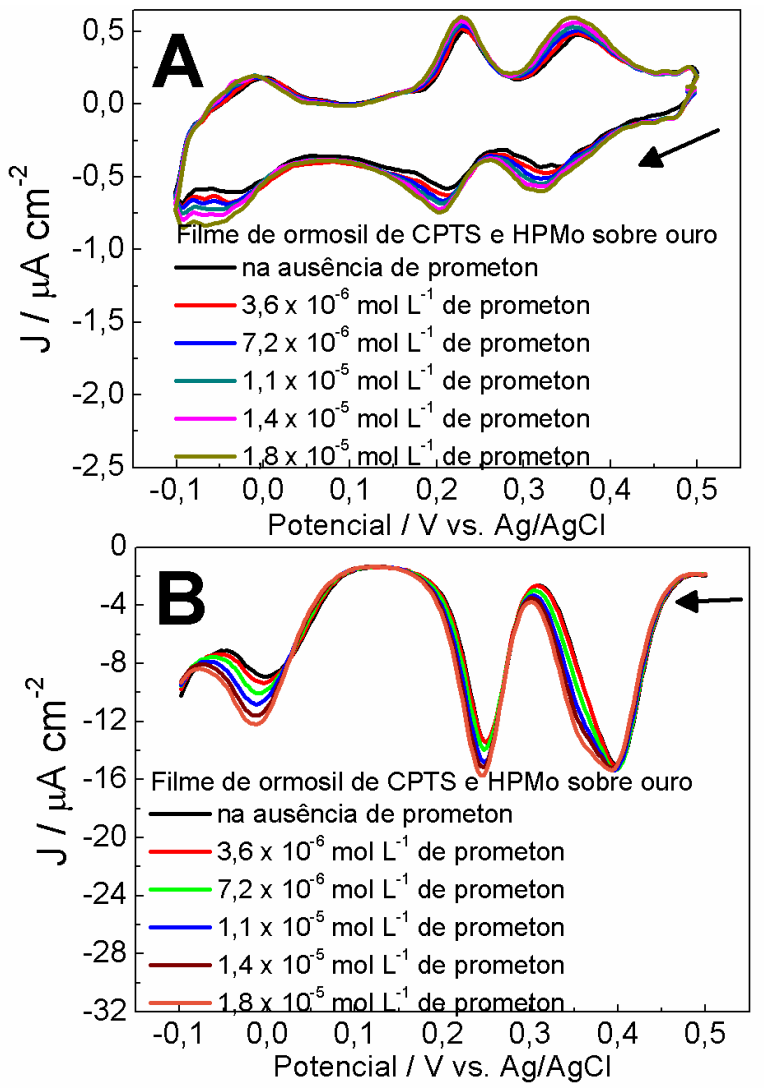

Figura 3: A) Voltamogramas cíclicos para o eletrodo de ouro modificado com o filme híbrido do ormosil de CPTS e HPMo na ausência e na presença de diferentes concentrações de prometon em meio ácido (pH 1,0). A velocidade de varredura utilizada foi de $20 \mathrm{mV} \mathrm{s}^{-1}$. B) Voltamogramas de pulso diferencial para o eletrodo de ouro modificado com o filme híbrido do ormosil de CPTS e HPMo na ausência e na presença de diferentes concentrações de prometon em meio ácido $(\mathrm{pH} 1,0)$.

SREEDHAR et al. [25] obtiveram um limite de detecção de $2 \times 10^{-8} \mathrm{~mol} \mathrm{~L}^{-1}$ para prometon usando eletrodo gotejante de mercúrio em $\mathrm{pH}$ 4,0 através de voltametria de pulso diferencial. No entanto, o potencial de redução analisado foi muito negativo e da ordem de $-1,0 \mathrm{~V}$ vs. $\mathrm{Ag} / \mathrm{AgCl}$. Neste referido trabalho ainda houve uma proposta mecanística para a redução do prometon. A primeira etapa envolveria a protonação do nitrogênio sem substituinte seguido pela clivagem das ligações $\mathrm{C}-\mathrm{O}$ liberando - $\mathrm{CHO}$ e posteriormente com a protonação, $-\mathrm{CH}_{3} \mathrm{OH}$ promovendo a abertura do anel triazínico. A figura 4 mostra a diferença entre os valores de densidade de corrente para o filme de ormosil de CPTS e HPMo na presença e na ausência de prometon $(\Delta \mathrm{J})$ para cada voltamograma de pulso diferencial da figura $3 \mathrm{~B}$ na respectiva concentração testada. A partir da inclinação da reta desta figura, a sensibilidade do filme para prometon foi de $1,4 \times 10^{-7} \mu \mathrm{A} \mathrm{cm}^{-2} \mathrm{~mol}^{-}$ ${ }^{1}$ L. O limite de detecção para prometon foi obtido multiplicando por três o desvio padrão da densidade de corrente do filme de ormosil de CPTS e HPMo na ausência de prometon, resultando em 3,5 x $10^{-6} \mathrm{~mol} \mathrm{~L}^{-1}$. Desta forma, apesar do limite de detecção obtido aqui para prometon ser maior que o encontrado na literatura, o potencial encontrado aqui para a eletroredução do herbicida pelo filme de CPTS e HPMo foi de 0,0 V vs. $\mathrm{Ag} / \mathrm{AgCl}$, portanto termodinamicamente mais favorável quando comparado a outros trabalhos da literatura $[24,25]$ pois o potencial necessário para a redução é menor. 


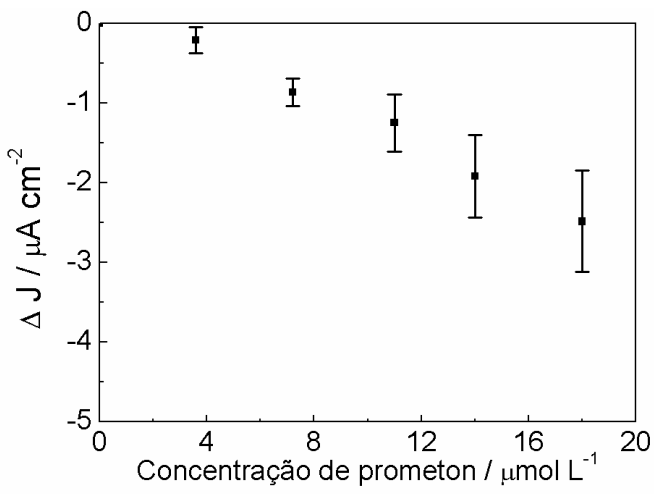

Figura 4: Variação da densidade de corrente $(\Delta \mathrm{J})$ em função da concentração de prometon.

A figura 5 mostra o espectro de FTIR para uma pastilha de $\mathrm{KBr}$ contendo o ormosil de CPTS e HPMo. Aquelas bandas que estão baixo de $900 \mathrm{~cm}^{-1}$ são atribuídas aos estiramentos assimétricos das tríades $\mathrm{M}_{3} \mathrm{O}_{13}$ do POM HPMo. A banda que surgiu em $957 \mathrm{~cm}^{-1}$ é atribuída ao estiramento assimétrico Mo- $\mathrm{O}_{\mathrm{t}}$, onde $\mathrm{O}_{\mathrm{t}}$ é um átomo de oxigênio terminal na estrutura Keggin do POM. A banda referente ao estiramento $-\mathrm{CN}$ do CPTS na rede do ormosil apareceu em $1622 \mathrm{~cm}^{-1}$. A região do espectro compreendida entre 1067 e $1155 \mathrm{~cm}^{-1}$ é uma região de sobreposição de bandas. Neste intervalo, os seguintes modos vibracionais podem estar presentes: acoplamento entre os estiramentos assimétricos de Mo-Ot e P-O e, também, aos estiramentos assimétricos SiO-C e Si-O-Si da rede de ormosil. A banda larga em $3400 \mathrm{~cm}^{-1}$ é atribuída aos estiramentos $-\mathrm{OH}[\underline{14}, \underline{26}$. Não foi possível identificar interações moleculares através do espectro pois não foi constatado deslocamento ou alargamento de banda (s) [27]. Recentemente, nosso grupo de pesquisa conseguiu identificar através do espectro de FTIR que a interação entre HPMo e um ormosil contendo grupos metacrilato ocorre via ligações de hidrogênio devido ao deslocamento da banda do grupo carbonila para menor valor de número de onda [2] $]$.

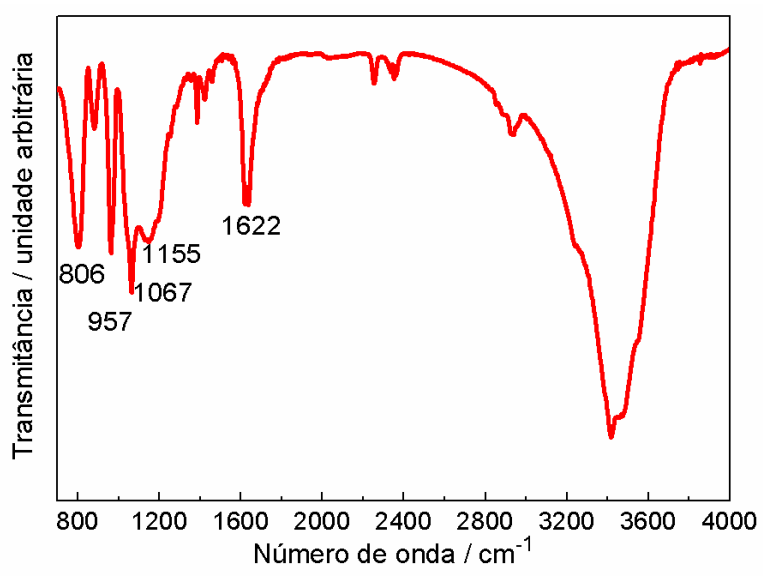

Figura 5: Espectro de FTIR na forma de pastilha de KBr para o ormosil de CPTS e HPMo.

\section{CONCLUSÃO}

Neste trabalho, o filme híbrido do ormosil de CPTS e HPMo depositado em eletrodo de ouro foi capaz de eletroreduzir o herbicida triazínico prometon em um potencial de $0,0 \mathrm{~V}$ vs. $\mathrm{Ag} / \mathrm{AgCl}$ em meio ácido $(\mathrm{pH} 1,0)$ conforme observado pelas análises de voltametria de pulso diferencial. A faixa de concentração na qual tal processo ocorreu foi de $3,6 \times 10^{-6}$ a $18 \times 10^{-6}$ mol L ${ }^{-1}$. O potencial para a eletroredução de prometon encontrado aqui é consideravelmente menor que os valores encontrados na literatura $(+0,75 \mathrm{~V}$ ou $-1,0 \mathrm{~V}$ vs. $\mathrm{Ag} / \mathrm{AgCl}$ ), o que significa que uma energia menor é necessária para realizar esta prática. Não foi possível identificar algum tipo de interação molecular através de espectroscopia de absorção na região do infravermelho. Com as contribuições apresentadas aqui, espera-se que o filme híbrido estudado possa auxiliar em aplicações na área ambiental, como por exemplo, um material a ser usado para reconhecer a presença de herbicidas triazínicos em água. 


\section{AGRADECIMENTOS}

ALS agradece ao Conselho Nacional de Desenvolvimento Científico e Tecnológico (CNPq) (Processo: 427243/2016-0) e à Fundação de Amparo à Pesquisa do Estado de São Paulo (FAPESP) (Processo: 2015/24136-4) pelo auxílio financeiro. VOM e JHP agradecem à FAPESP por suas bolsas de iniciação científica, (Processo: 2018/05200-1) e (Processo: 2016/21616-8), respectivamente. KRF agradece à FAPESP pelo apoio financeiro (Processo: 2017/20006-4). ALS agradece ao Laboratório de Microfabricação (LMF) do Laboratório Nacional de Nanotecnologia (LNNano) pela preparação das lâminas de ouro.

\section{BIBLIOGRAFIA}

[1] YUAN, Y., YAN, G.H., HUANG, S.H., et al., "Preparation of hydrophobic SiO2/PMHS sol and ORMOSIL antireflective films for solar glass cover", Solar Energy, v. 130, pp. 1-9, 2016.

[2] SANCHEZ, C., BOISSIERE, S., CASSAIGNON, C., et al., "Molecular Engineering of Functional Inorganic and Hybrid Materials", Chemistry of Materials, v. 26, n. 1, pp. 221-238, 2013.

[3] JOSÉ, N.M., SANCHEZ DE ALMEIDA PRADO, L.A., "Materiais híbridos orgânico-inorgânicos: Preparação e algumas aplicações", Química Nova, v. 28, n. 2, pp. 281-288, 2005.

[4] ALFAYA, A.A.S.; KUBOTA, L.T., "A utilização de materiais obtidos pelo processo de sol-gel na construção de biossensores”, Química Nova, v. 25, n. 5, pp. 835-841, 2002.

[5] PARDO, R., ZAYAT, M., LEVY, D., "Photochromic organic-inorganic hybrid materials", Chemical Society Reviews, v. 40, n. 2, pp. 672-687, 2011.

[6] CALDARA, M., COLLEONI, C., GUIDO, E., et al., "Optical monitoring of sweat pH by a textile fabric wearable sensor based on covalently bonded litmus-3-glycidoxypropyltrimethoxysilane coating", Sensors and Actuators, B: Chemical, v. 222, pp. 213-220, 2016.

[7] DE OLIVEIRA, M., LOPES DE SOUZA, A., SCHNEIDER, J., et al., "Local structure and photochromic response in ormosils containing dodecatungstophosphoric acid", Chemistry of Materials, v. 23, n. 4, pp. 953963, 2011.

[8] WANG, M.S., XU, G., ZHANG, Z.-J., et al., "Inorganic-organic hybrid photochromic materials", Chemical Communications, v. 46, n. 3, pp. 361-376, 2010.

[9] WALSH, J.J., BOND, A.M., FORSTER, R.J., et al., "Hybrid polyoxometalate materials for photo(electro-) chemical applications", Coordination Chemistry Reviews, v. 306, pp. 217-234, 2016.

[10] SADAKANE, M., STECKHAN, E., "Electrochemical Properties of Polyoxometalates as Electrocatalysts", Chemical Reviews, v. 98, pp. 219-237, 1998.

[11] CHEN, L., CHEN, W.-L., WANG, X.-L., et al., "Polyoxometalates in dye-sensitized solar cells", Chemical Society Reviews, v. 48, pp. 260-284, 2019.

[12] HISKIA, A., MYLONAS, A., PAPACONSTANTINOU, E., "Comparison of the photoredox properties of polyoxometallates and semiconducting particles", Chemical Society Reviews, v. 30, n. 1, pp. 62-69, 2001.

[13] SOUZA, A.L., TREMILIOSI-FILHO, G., KUBOTA, L.T., et al., "Poly(dimethylsiloxane) as a precoating in layer-by-layer films containing phosphotungstate nanoclusters electrochemically sensitive toward s-triazines", RSC Advances, v. 4, n. 56, pp. 29612, 2014.

[14] OLÍVIO, P.H.P., CORREIRA, L.C., PAULA, J.H., et al., "Exploring electrochemical reactivity toward ametryn of hybrid silicate films with phosphomolybdic acid", Materials Science and Engineering B: SolidState Materials for Advanced Technology, v. 229, pp. 13-19, 2018.

[15] ZULKIFLI, S.N., RAHIM, H.A., LAU, W.J., "Detection of contaminants in water supply: A review on state-of-the-art monitoring technologies and their applications", Sensors and Actuators, B: Chemical, v. 255, pp. 2657-2689, 2018.

[16] FERNÁNDEZ-RAMOS, C., FERRER, I., MAUCH, K., et al., "Identification of prometon, deisopropylprometon, and hydroxyprometon in groundwater by high resolution liquid chromatography/mass spectrometry", Science of the Total Environment, v. 497-498, pp. 459-466, 2014.

[17] LIANG, L., WANG, X., SUN, Y., et al., "Magnetic solid-phase extraction of triazine herbicides from rice using metal-organic framework MIL-101(Cr) functionalized magnetic particles", Talanta, v. 179, pp. 512-519, 2018.

[18] SCOGNAMIGLIO, V., PEZZOTTI, I., PEZZOTTI, G., et al., "A new embedded biosensor platform based on micro-electrodes array (MEA) technology", Sensors and Actuators, B: Chemical, v. 176, pp. 275283, 2013. 
[19] MCGARVEY, G.B., MOFFAT, J.B., "A study of solution species generated during the formation of 12 heteropoly oxometalate catalysts", Journal of Molecular Catalysis, v. 69, n. 1, pp. 137-155, 1991.

[20] TANAKA, N., UNOURA, K.E.I., ITABASHI, E., "Voltammetric and Spectroelectrochemical Studies of 12 -Molybdophosphoric Acid in Aqueous and Water-Dioxane Solutions at a Gold-Minigrid Optically Transparent Thin-Layer Electrode", Inorganic Chemistry, v.21, n. 4, pp. 1662-1666, 1982.

[21] LI, Z., CHEN, J., PAN, D., et al., "A sensitive amperometric bromate sensor based on multi-walled carbon nanotubes/phosphomolybdic acid composite film”, Electrochimica Acta, v. 51, n. 20, pp. 4255-4261, 2006.

[22] RAHMAN, M.A., WON, M.S., WEI, P.H., et al., "Electrochemical detection of ClO3-, BrO3-, and IO3at a phosphomolybdic acid linked 3-aminopropyl-trimethoxysilane modified electrode", Electroanalysis, v. 18 , n. 10, pp. 993-1000, 2006.,m

[23] ROHLFING, D.F., RATHOUSKY, J., ROHLFING, Y., et al., "Functionalized mesoporous silica films as a matrix for anchoring electrochemically active guests", Langmuir, v. 21, n. 24, pp. 11320-11329, 2005.

[24] OLIVEIRA-BRETT, A.M., DA SILVA, L.A., "A DNA-electrochemical biosensor for screening environmental damage caused by s-triazine derivatives”, Analytical and Bioanalytical Chemistry, v. 373, pp. 717-723, 2002.

[25] SREEDHAR, M., REDDY, T.M., BALAJI, K., et al., "Electrochemical reduction behavior and polarographic determination of methoxy triazine herbicides in environmental samples", Analytical Letters, v. 43, pp. 674-686, 2010.

[26] SOCRATES, G., Infrared and Raman characteristic group frequencies, 3 ed., New York, John Wiley \& Sons, 2001.

[27] PAVINATTO, A., SOUZA, A.L., DELEZUK, J.A.M., et al., "Interaction of O-acylated chitosans with biomembrane models: Probing the effects from hydrophobic interactions and hydrogen bonding", Colloids and Surfaces B: Biointerfaces, v. 114, pp. 53-59, 2014.

[28] CORREIA, L.A., MARGARIDO, V.O., SOUZA, A.L., "Performance eletrocatalítica frente a nitrito de um filme híbrido de fosfomolibdato esílica organomodificada", Revista Matéria, v. 24, n. 04, pp. e-12491, 2019.

\section{ORCID}

Victória de Oliveira Margarido Julia Helena de Paula Kelly Roberta Francisco https://orcid.org/0000-0002-7100-9868 https://orcid.org/0000-0003-1473-8762 Adriano Lopes de Souza https://orcid.org/0000-0001-6673-3280 https://orcid.org/0000-0001-6593-8399 